\title{
Absolutely continuous invariant measures for some piecewise hyperbolic affine maps
}

\author{
Tomas Persson \\ Centre for Mathematical Sciences, Department of Mathematics, \\ Lund Institute of Technology, P.O. Box 118, SE-22100 Lund, Sweden \\ tomasp@maths.1th.se, http://www.maths.1th.se/ ^ tomasp
}

June 28, 2018

\begin{abstract}
A class of piecewise affine hyperbolic maps on a bounded subset of the plane is considered. It is shown that if a map from this class is sufficiently area-expanding then almost surely this map has an absolutely continuous invariant measure.
\end{abstract}

Acknowledgement. The author is very grateful to Jörg Schmeling for interesting and useful discussions.

\section{Introduction}

In 14, Pesin studied a general class of piecewise diffeomorphisms with a hyperbolic attractor. He showed the existence of the Sinai-Bowen-Ruelle measure, or SBR-measure for short, and studied the ergodic properties of this measure. If $f: M \rightarrow M$ is the system in question then the SBR-measure is a weak limit point of the sequence of measures

$$
\mu_{n}=\frac{1}{n} \sum_{k=0}^{n-1} \nu \circ f^{-k},
$$

where $\nu$ denotes the Lebesgue measure. Pesin showed that the SBR-measure has at most countably many ergodic components. This measure is the physically relevant measure as it captures the behaviour of the orbits of points from a set of positive Lebesgue measure.

For a more restricted class, Sataev [15] showed that there are only finitely many ergodic components. Schmeling and Troubetzkoy studied in 17] a more general class than Pesin's and proved the existence of the SBR-measure. Their method to deal with the non-invertibility of the system was to lift the system to a higher dimension and get an invertible system on which the calculations were made. In this way methods from invertible systems could be used. The result could then be projected back to the original system.

In 1, Alexander and Yorke considered a one parameter class of maps called the fat baker's transformations. These maps are piecewise affine maps of the 
square with one expanding and one contracting direction. Their results together with the result of Solomyak in 18, imply that for a positive measure set of parameters, there is an absolutely continuous invariant measure.

The Belykh map, was first introduced in [2] by Belykh. Schmeling and Troubetzkoy considered in [17] the Belykh map for a wider range of parameters. The fat baker's transformations are a special case of the Belykh map in this wider range of parameters. The Belykh map was further investigated in [16].

In this article we consider a class of piecewise affine hyperbolic maps on a set $K \subset \mathbb{R}^{2}$, with one contracting and one expanding direction. This class is contained in the class of maps studied in [17 and it contains the Belykh maps as well as the fat baker's transformations.

It is shown that if a functions from this class is sufficiently area-expanding then almost surely (in the sense of Corollary 1) there is an absolutely continuous invariant measure. The method used to show this is a development of the method from [12. Here a new problem arises: The symbolic space changes as the parameters changes. In this paper a way to handle this problem is introduced. The different symbolic spaces are embedded in a larger space and certain estimates are carried out that makes this larger space possible to handle.

In [21] and [22], Tsujii considered two classes of maps in two dimension and showed that almost all of these maps have absolutely continuous invariant measure. These two classes are different from the class of maps considered in this paper. Tsujii also used the method from [12, but in a different way than is used in this paper.

Similar results in two dimensions, but in the case of expanding maps, were independently obtained by Buzzi in [5] and Tsujii in [19. The corresponding results for arbitrary dimension are in [4] and [20].

\section{A class of piecewise hyperbolic maps}

Let $K \subset \mathbb{R}^{2}$ be compact and connected. Assume that $K$ can be decomposed according to

$$
K=\bigcup_{i=1}^{a} \bar{K}_{i}
$$

where each $K_{i}$ is an open and non-empty set with the boundary consisting of finitely many $C^{2}$ curves. Thus, there are closed $C^{2}$ curves $N_{i}$ and $M_{i}$ such that

$$
\bigcup_{i=1}^{a} \partial K_{i}=\left(\bigcup_{i=1}^{b} N_{i}\right) \cap\left(\bigcup_{i=1}^{c} M_{i}\right), \partial K=\bigcup_{i=1}^{c} M_{i} .
$$

Let $\mathcal{Z}=\left\{K_{i}\right\}$ denote the partion of $K$.

Assume that the sets $N_{i} \cap N_{j}, M_{i} \cap M_{j}$ and $N_{i} \cap M_{k}$ consists of finitely many points if $i \neq j$, and there exists a constant $H$ such that if $\left(t_{1}, t_{2}\right) \in T_{p} N_{i j}$ then $\left|t_{2} / t_{1}\right|<H$. Let $N=\cup N_{i}$ and $M=\cup M_{i}$.

See Figure 1 for an example of $K, N_{i}$ and $M_{i}$.

Consider maps $f: K \backslash N \rightarrow K$ that satisfy the following two conditions, (A1) and (A2).

(A1) There are numbers $\lambda_{1}, \ldots, \lambda_{a}<1<\gamma_{1}, \ldots, \gamma_{a}$ and $u_{1}, \ldots u_{a}, v_{1}, \ldots v_{a} \in \mathbb{R}$ with $u_{i} \neq u_{j}$ whenever $i \neq j$, such that for any $i=1, \ldots, a$ the map $f$ 


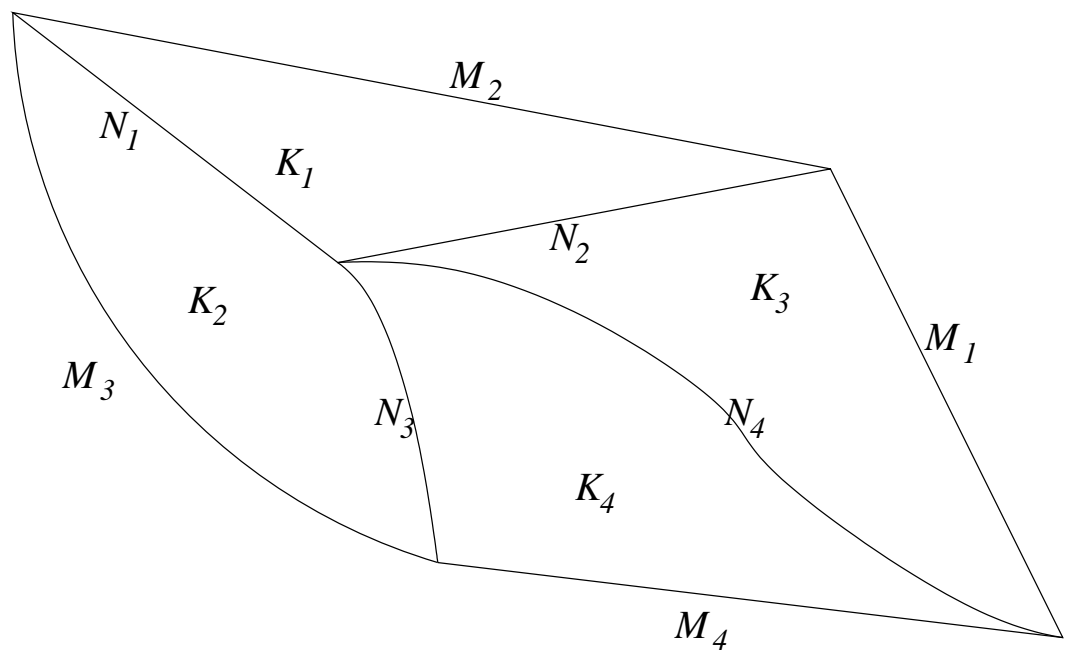

Figure 1: An example of the domain $K$.

restricted to $K_{i}$ is defined by

$$
\left.f\right|_{K_{i}}\left(x_{1}, x_{2}\right)=f_{i}\left(x_{1}, x_{2}\right)=\left(\lambda_{i} x_{1}+u_{i}, \gamma_{i} x_{2}+v_{i}\right) .
$$

The notation $f_{\bar{\lambda}, \bar{\gamma}, \bar{u}, \bar{v}}$ for $f$ will be used to emphasise the dependence on the parameters $\bar{\lambda}=\left(\lambda_{1}, \ldots, \lambda_{a}\right), \bar{\gamma}=\left(\gamma_{1}, \ldots, \gamma_{a}\right), \bar{u}=\left(u_{1}, \ldots u_{a}\right)$ and $\bar{v}=$ $\left(v_{1}, \ldots v_{a}\right)$.

Let $\mathcal{Z}_{0}=\mathcal{Z}$ and assume that $\mathcal{Z}_{k}=\left\{K_{i}^{(k)}\right\}_{i=1}^{a_{k}}$ is defined. (Note that $a_{0}=a$.) Then define the partition $\mathcal{Z}_{k+1}=\left\{K_{i}^{(k+1)}\right\}_{i=1}^{a_{k+1}}$ by

$$
\mathcal{Z}_{k+1}=\mathcal{Z}_{k} \wedge\left\{f\left(K_{i}^{(k)}\right)\right\}_{i=1}^{a_{k}} .
$$

The set $\bigcup_{i=1}^{a_{k}} K_{i}^{\left(a_{k}\right)}$ is the set of points $x \in K$ such that for each $l=0,1, \ldots, k$ the point $f^{l}(x)$ is defined and $f^{l}(x) \notin N \cup M$.

Since each $K_{i}$ has piecewise $C^{2}$ boundary so has each $K_{j}^{(k)}$. There are thus closed $C^{2}$ curves $N_{i}^{(k)}$ such that

$$
\bigcup_{i=1}^{a_{k}} \partial K_{i}^{(k)}=\bigcup_{i=1}^{b_{k}} N_{i}^{(k)}
$$

and $N_{i}^{(k)} \cap N_{j}^{(k)}$ is a finite set if $i \neq j$.

(A2) There is a number $\tau \geq 1$ such that $\left(\min \gamma_{i}\right)^{\tau}>D_{\tau}+1$ where

$$
D_{\tau}=\max \left\{\# A \mid A \subseteq\left\{1,2, \ldots, b_{\tau}\right\} \text { s.t. } \bigcap_{i \in A} N_{i}^{(\tau)} \neq \emptyset\right\} .
$$

That is, $D_{\tau}$ is the maximal number of lines from $\left\{N_{i}^{(\tau)}\right\}$ that crosses at one point. The number $D_{\tau}$ is finite since the set $\left\{N_{i}^{(\tau)}\right\}$ is finite.

Remark. Condition (A2) implies that the multiplicity entropy (see [3] and [10] for a definition) is less than the positive Lyapunov exponent. 


\section{The results}

We are going to prove the following theorem.

Theorem 1. Assume that for all $t \in I=\left(t_{0}, t_{1}\right)$, the maps $f_{t \bar{\lambda}, \bar{\gamma}, \bar{u}, \bar{u}}$ satisfy the conditions (A1) and (A2) with a uniform $\tau$. If

$$
\frac{t_{0} \min \left\{\lambda_{i}\right\} \min \left\{\gamma_{i}^{2}\right\}}{\max \left\{\gamma_{i}\right\}}>1,
$$

and one of the following conditions are satisfied

$$
\begin{array}{ll}
\text { 1. } & t_{1} \lambda_{\max }<0.5, \quad \frac{\max \left\{\left|u_{i}-u_{j}\right|,\left|u_{i}\right|\right\}}{\min \left\{\left|u_{i}-u_{j}\right|: u_{i} \neq u_{j}\right\}}<\frac{1-t_{1} \lambda_{\max }}{t_{1} \lambda_{\max }-2\left(t_{1} \lambda_{\max }\right)^{3}}, \\
\text { 2. } t_{1} \lambda_{\max }<0.61, \quad \frac{\max \left\{\left|u_{i}-u_{j}\right|,\left|u_{i}\right|\right\}}{\min \left\{\left|u_{i}-u_{j}\right|: u_{i} \neq u_{j}\right\}}<\frac{1-t_{1} \lambda_{\max }}{t_{1} \lambda_{\max }-2\left(t_{1} \lambda_{\max }\right)^{4}}, \\
\text { 3. } t_{1} \lambda_{\max }<0.68, \quad \frac{\max \left\{\left|u_{i}-u_{j}\right|,\left|u_{i}\right|\right\}}{\min \left\{\left|u_{i}-u_{j}\right|: u_{i} \neq u_{j}\right\}}<\frac{1-t_{1} \lambda_{\max }}{t_{1} \lambda_{\max }-2\left(t_{1} \lambda_{\max }\right)^{5}},
\end{array}
$$

where $\lambda_{\max }=\max \left\{\lambda_{i}\right\}$, then for almost every $t \in I$ there exists an $f_{t \bar{\lambda}, \bar{\gamma}, \bar{u}, \bar{v}^{-}}$ invariant measure, absolutely continuous with respect to Lebesgue measure.

Conclude the following

Corollary 1. Let $P$ be the set of parameters $(\bar{\lambda}, \bar{\gamma}, \bar{u}, \bar{v})$ such that

$$
\frac{\min \left\{\lambda_{i}\right\} \min \left\{\gamma_{i}^{2}\right\}}{\max \left\{\gamma_{i}\right\}}>1
$$

and one of the following conditions are satisfied

$$
\begin{aligned}
& \text { 1. } \max \left\{\lambda_{i}\right\}<0.5, \quad \frac{\max \left\{\left|u_{i}-u_{j}\right|,\left|u_{i}\right|\right\}}{\min \left\{\left|u_{i}-u_{j}\right|: u_{i} \neq u_{j}\right\}}<\frac{1-\max \left\{\lambda_{i}\right\}}{\max \left\{\lambda_{i}\right\}-2\left(\max \left\{\lambda_{i}\right\}\right)^{3}}, \\
& \text { 2. } \max \left\{\lambda_{i}\right\}<0.61, \frac{\max \left\{\left|u_{i}-u_{j}\right|,\left|u_{i}\right|\right\}}{\min \left\{\left|u_{i}-u_{j}\right|: u_{i} \neq u_{j}\right\}}<\frac{1-\max \left\{\lambda_{i}\right\}}{\max \left\{\lambda_{i}\right\}-2\left(\max \left\{\lambda_{i}\right\}\right)^{4}}, \\
& \text { 3. } \max \left\{\lambda_{i}\right\}<0.68, \quad \frac{\max \left\{\left|u_{i}-u_{j}\right|,\left|u_{i}\right|\right\}}{\min \left\{\left|u_{i}-u_{j}\right|: u_{i} \neq u_{j}\right\}}<\frac{1-\max \left\{\lambda_{i}\right\}}{\max \left\{\lambda_{i}\right\}-2\left(\max \left\{\lambda_{i}\right\}\right)^{5}} .
\end{aligned}
$$

If $f_{\bar{\lambda}, \bar{\gamma}, \bar{u}, \bar{u}}$ satisfies the conditions (A1) and (A2) then for Lebesgue almost every $(\bar{\lambda}, \bar{\gamma}, \bar{u}, \bar{v}) \in P$, there is an $f_{\bar{\lambda}, \bar{\gamma}, \bar{u}, \bar{v}}$-invariant measure, absolutely continuous with respect to Lebesgue measure.

\section{A condition on transversality for power series}

The corollary of the following lemma will be used to prove Theorem 1 The Lemma appears in a somewhat less general form in 12. The proof from [12] works here as well.

Lemma 1. Let $C \geq 1$. Then there is a constant $\delta>0$ such that for any function $g$ of the form

$$
g(x)=1+\sum_{k=1}^{\infty} b_{k} x^{k}, b_{k} \in[-C, C]
$$


the following implication holds true for $n=2,3,4$

$$
g(x) \leq \delta, x \in\left(0, Q_{n}\right), C<f_{n}(x) \Longrightarrow g^{\prime}(x) \leq-\delta,
$$

where $f_{n}(x)=\frac{1-x}{x-2 x^{n+1}}, Q_{2}=0.5, Q_{3}=0.61$ and $Q_{4}=0.68$.

Proof. Let $h_{n}(x)=1-C \sum_{k=1}^{n} x^{k}+C \sum_{k=n+1}^{\infty} x^{k}=1-C \frac{x-2 x^{n+1}}{1-x}$ and $f_{n}(x)=$ $\frac{1-x}{x-2 x^{n+1}}$. Let $Q_{2}=0.5, Q_{3}=0.61$ and $Q_{4}=0.68$. This implies that there is a $\delta>0$ such that for $n=2,3,4$

i) $h_{n}(x)>\delta$ if $C<f_{n}(x)$

ii) $h_{n}^{\prime}(x)<-\delta$ if $x \in\left(0, Q_{n}\right)$.

Thus $h_{n}(x)>\delta$ and $h_{n}^{\prime}(x)<-\delta$ provided $x<Q_{n}$ and $C<f_{n}(x)$.

Let $g(x)$ be of the form (11). There are $c_{k} \geq 0$ such that the function $G_{n}(x)=g(x)-h_{n}(x)$ can be written as $G_{n}(x)=\sum_{k=1}^{n} c_{k} x^{k}-\sum_{k=n+1}^{\infty} c_{k} x^{k}$. By the above mentioned properties of $h_{n}$ we have for any $x \in\left(0, Q_{n}\right), C<f_{n}(x)$

$$
g(x)<\delta \Rightarrow G_{n}(x)<0 \Rightarrow G_{n}^{\prime}(x)<0 \Rightarrow g^{\prime}(x)<-\delta .
$$

The second implication is proved by

$$
\begin{aligned}
G_{n}(x)<0 \Rightarrow \sum_{k=1}^{n} c_{k} x^{k}< & \sum_{k=n+1}^{\infty} c_{k} x^{k} \Rightarrow \\
& \sum_{k=1}^{\infty} k c_{k} x^{k}<\sum_{k=n+1}^{\infty} k c_{k} x^{k} \Rightarrow G_{n}^{\prime}(x)<0 .
\end{aligned}
$$

Corollary 2. Let $n \in\{2,3,4\}$ and let $Q_{n}$ and $f_{n}$ be as in Lemma 1 . Let $s_{k}$ be a sequence with $s_{k} \in[-C, C]$. Then for any $l>0$ the set

$$
\left\{q \in\left(q_{0}, Q_{n}\right)\left|C<f_{n}(q),\right| q^{l}+\sum_{k=l+1}^{\infty} s_{k} q^{k} \mid<r\right\}
$$

is contained in an interval of length at most $2 \delta^{-1} q_{0}^{-l} r$.

Proof. Note that $\left|q^{l}+\sum_{k=l+1}^{\infty} s_{k} q^{k}\right|<r$ implies $\left|1+\sum_{k=l+1}^{\infty} s_{k} q^{k-l}\right|<r q_{0}^{-l}$ if $q \geq q_{0}$. Lemma 11 implies that on the set $\left\{q \in\left(0, Q_{n}\right) \mid C<f_{n}(q)\right\}$ the graph of the function $q \mapsto 1+\sum_{k=l+1}^{\infty} s_{k} q^{k-l}$ crosses 0 transversally in at most one point and the slope is at most $-\delta$ around this point. Hence $\left|1+\sum_{k=l+1}^{\infty} s_{k} q^{k-l}\right|<r q_{0}^{-l}$ on an interval of length not more than $2 \delta^{-1} q_{0}^{-l} r$ and therefore $\left|q^{l}+\sum_{k=l+1}^{\infty} s_{k} q^{k}\right|<r$ can only hold in this interval.

\section{Proof of Theorem 1}

We will use the method from [16] to prove Theorem 1 This method is based on [12]. The idea is to integrate the density of the measure and then integrate with respect to the parameter. If this integral is finite then almost surely the density is integrable and so the measure is absolutely continuous with respect to Lebesgue measure. To prove that this is the case it is necessary to control how the measure changes with the parameter.

We begin with some notation and general theory and then make the estimates needed later in the proof. 


\subsection{Notation and general theory}

Fix $\bar{\lambda}, \bar{\gamma}, \bar{u}$ and $\bar{v}$. Let $\lambda_{\min }=\min \left\{\lambda_{i}\right\}, \lambda_{\max }=\max \left\{\lambda_{i}\right\}, \gamma_{\min }=\min \left\{\gamma_{i}\right\}$ and $\gamma_{\max }=\max \left\{\gamma_{i}\right\}$. We will use the shorter notation $f_{t}$ to denote $f_{t \bar{\lambda}, \bar{\gamma}, \bar{u}, \bar{u}}$.

Let $\hat{K}=K \times[0,1]$ and $\hat{K}_{i}=K_{i} \times[0,1]$. The sets $\hat{N}, \hat{M}, \ldots$ are defined analogously. We use the idea from [17] and lift the map $f_{t}$ to an injective map $\hat{f}_{t}$ on $\hat{K}$ by

$$
\left.\hat{f}_{t}\right|_{\hat{K}_{i}}\left(x_{1}, x_{2}, x_{3}\right)=\left(f_{t}\left(x_{1}, x_{2}\right), \theta x_{3}+i /(a+1)\right),
$$

where $0<\theta<1 /(a+1)$. The map $\pi: \hat{K} \rightarrow K,\left(x_{1}, x_{2}, x_{3}\right) \mapsto\left(x_{1}, x_{2}\right)$ is the projection of $\hat{K}$ on $K$. It satisfies $\pi\left(\hat{f}_{t}\left(x_{1}, x_{2}, x_{3}\right)\right)=f_{t}\left(\pi\left(x_{1}, x_{2}, x_{3}\right)\right)$.

Let $\hat{D}_{t}^{+}=\left\{\hat{p} \in \hat{K} \mid \hat{f}_{t}^{n}(\hat{p}) \notin \hat{N} \cup \hat{M}, \forall n \in \mathbb{N}\right\}$ and $\hat{D}_{t}=\bigcap_{n=0}^{\infty} \hat{f}_{t}^{n}\left(\hat{D}_{t}\right)$. The set $\hat{\Lambda}_{t}=\overline{\hat{D}}_{t}$ is the attractor of $\hat{f}_{t}$.

The condition (A2) is a more general version of condition (H9) in [14. It appears in [17. Theorem 6.1 in [17 can be applied to conclude that there are constants $c_{t}>0$ such that for any $\varepsilon>0$ and any $n \in \mathbb{N}$

$$
\hat{\nu}\left(\hat{f}_{t}^{-n}(U(\varepsilon, \hat{N} \cup \hat{M}))\right)<c_{t} \varepsilon
$$

where $U(\varepsilon, \hat{N} \cup \hat{M})$ denotes the $\varepsilon$-neighborhood of $\hat{N} \cup \hat{M}$. This shows that the functions $\hat{f}_{t}$ are in the class of functions from [14]. Moreover, the maps $f_{t}$ also satisfies the conditions in 17. This gives us the following results.

i) Let $V^{\mathrm{u}} \subset K$ be a curve in the unstable direction, i.e. there are numbers $\rho, \sigma_{1}$ and $\sigma_{2}$ such that $V^{\mathrm{u}}=\left\{\left(x_{1}, x_{2}\right) \in K \mid x_{1}=\rho, \sigma_{1}<x_{2}<\sigma_{2}\right\}$. Let $\hat{V}^{\mathrm{u}}=\pi^{-1}\left(V^{\mathrm{u}}\right)$ be the corresponding manifold in $\hat{K}$. Let $\nu_{V^{\mathrm{u}}}$ and $\hat{\nu}_{\hat{V} u}$ denote the normalised Lebesgue measure on $V^{\mathrm{u}}$ and $\hat{V}^{\mathrm{u}}$ respectively. The sequence of measures $\hat{\mu}_{n}^{t}=\frac{1}{n} \sum_{k=0}^{n-1} \hat{\nu}_{\hat{V}^{\mathrm{u}}} \circ \hat{f}_{t}^{-k}$ converges weakly to an SBR-measure $\hat{\mu}_{\mathrm{SBR}}^{t}$. The projection of this measure $\hat{\mu}_{\mathrm{SBR}}^{t} \circ \pi^{-1}$ is an SBR-measure for $f_{t}$ and we thus write $\mu_{\mathrm{SBR}}^{t}=\hat{\mu}_{\mathrm{SBR}}^{t} \circ \pi^{-1}$.

ii) Given $\varepsilon>0$, the set

$$
\hat{D}_{t, \varepsilon, c}=\left\{\hat{x} \in \hat{\Lambda}_{t} \mid d\left(\hat{f}_{t}^{n}(\hat{x}), \hat{N}\right) \geq c e^{-\varepsilon n}\right\}
$$

is non-empty if $c$ is sufficiently small and the set $\hat{D}_{t, \varepsilon}=\bigcup_{i=1}^{\infty} \hat{D}_{t, \varepsilon, i^{-1}}$ has full $\hat{\mu}_{\mathrm{SBR}}^{t}$-measure, $\hat{\mu}_{\mathrm{SBR}}^{t}\left(\hat{D}_{t, \varepsilon}\right)=1$.

iii) The conditional measures of $\hat{\mu}_{\mathrm{SBR}}^{t}$ on the unstable manifolds are absolutely continuous with respect to Lebesgue measure.

iv) The entropy of the measure $\hat{\mu}_{\mathrm{SBR}}^{t}$ is

$$
h_{\hat{\mu}_{\mathrm{SBR}}^{t}}=\int_{\hat{\Lambda}_{t}} \log \chi_{t}(\hat{x}) \mathrm{d} \hat{\mu}_{\mathrm{SBR}}^{t}(\hat{x}),
$$

where $\chi_{t}(\hat{x})$ is the positive Lyapunov exponent at the point $\hat{x}$ for the map $\hat{f}_{t}$. In particular, $\log \left(\gamma_{\min }\right) \leq h_{\hat{\mu}_{\mathrm{SBR}}^{t}} \leq \log \left(\gamma_{\max }\right)$.

v) The measure $\hat{\mu}_{\mathrm{SBR}}^{t}$ has at most countably many ergodic components. 
The results i) - v) make it possible to define stable manifolds for $\hat{\mu}_{\mathrm{SBR}^{-}}^{t}$ almost every $\hat{x} \in \hat{K}$. If $\hat{x}=\left(x_{1}, x_{2}, x_{3}\right) \in \hat{D}_{t,-\log \left(t \lambda_{\max }\right)}$ then there is a $c=c(\hat{x})$ such that $\hat{x} \in \hat{D}_{t,-\log \left(t \lambda_{\max }\right), c}$, that is $d\left(\hat{f}_{t}^{n}(\hat{x}), \hat{N}\right) \geq c\left(t \lambda_{\max }\right)^{n}$ for all $n \geq 0$. If $\hat{y}=\left(y_{1}, y_{2}, y_{3}\right) \in \hat{K}$ with $\left|y_{1}-x_{1}\right|<c$ and $y_{2}=x_{2}$ then

$$
\left|\hat{f}_{t}^{n}(\hat{y})-\hat{f}_{t}^{n}(\hat{x})\right|_{1} \leq\left|y_{1}-x_{1}\right|\left(t \lambda_{\max }\right)^{n}<c\left(t \lambda_{\max }\right)^{n} \leq d\left(\hat{f}_{t}^{n}(\hat{x}), \hat{N}\right),
$$

where $|\cdots|_{1}$ denotes the modulus of the difference in the first coordinate. Hence the points $\hat{f}_{t}^{n}(\hat{y})$ and $\hat{f}_{t}^{n}(\hat{x})$ are never separated by a discontinuity and we say $\hat{y}$ is in the stable manifold of $\hat{x}$. The stable manifold of $\hat{x}$ is thus defined to be the set

$$
\hat{W}^{t, \mathrm{~s}}(\hat{x})=\left\{\hat{y} \in \hat{K}|| x_{1}-y_{1} \mid<c, y_{2}=x_{2}\right\},
$$

where $c$ is the largest constant such that $\hat{x} \in \hat{D}_{t,-\log \left(t \lambda_{\max }\right), c}$. This defines the stable manifold of $\hat{\mu}_{\mathrm{SBR}}^{t}$-a.e. point $\hat{x} \in \hat{K}$ since $\hat{\mu}_{\mathrm{SBR}}^{t}\left(\hat{D}_{t, \log \left(t \lambda_{\max }\right)}\right)=1$.

The stable manifold $W^{t, \mathrm{~s}}(x)$ is defined as the projection of corresponding stable manifold in $\hat{K}$. All the stable manifolds will therefore be parallel line segments but the length of the manifolds are only measurable ([14]).

Similarly the unstable manifolds can be defined. They consist of parallel line segments, orthogonal to the stable manifolds, and their length is measurable.

The partition of $K$ into stable manifolds is thus measurable and the conditional measures on these manifolds can be defined. Take $x \in \Lambda=\pi(\hat{\Lambda})$ and let $\mu_{\mathrm{SBR}}^{t, \mathrm{~s}, x}$ denote the conditional measure on the stable manifold $W^{t, \mathrm{~s}}(x)$.

The sequence of measures $\hat{\mu}_{n}^{t}$ may converge to a measure which is not ergodic, but if $\hat{V}^{\mathrm{u}} \subseteq \hat{W}^{t, \mathrm{u}}(\hat{x})$ for some $\hat{x}$ then $\hat{\mu}_{n}^{t}$ will converge to a unique ergodic component. To get control over $\hat{\mu}_{\mathrm{SBR}}^{t}$ for almost all $t \in I$ argue as follows. Since the size of the unstable manifolds depends only measurable on $t$ there is no $\hat{V}^{\mathrm{u}}$ that is contained in an unstable manifold for every $t \in I$. However, given $\varepsilon_{0}>0$ there is a set $J_{0} \subset I$ with $\nu\left(I \backslash J_{0}\right)<\varepsilon_{0}$ and a set $\hat{V}^{\mathrm{u}}$, independent of $t$, such that $\hat{V}^{\mathrm{u}} \subseteq \hat{W}^{t, \mathrm{u}}(\hat{x})$ for some $\hat{x}$ whenever $t \in J_{0}$. This construction has the following consequence. For any $t \in J_{0}$ the measure $\hat{\mu}_{n}^{t}$ converges to an ergodic measure and it is not necessary to take a subsequence. Indeed, if it is necessary to take a subsequence then there exists a set $A$ such that

$$
\liminf _{n \rightarrow \infty} \hat{\mu}_{n}^{t}(A)<\limsup _{n \rightarrow \infty} \hat{\mu}_{n}^{t}(A) .
$$

But this would contradict that $\hat{\mu}_{n}^{t}$ converges to a unique ergodic component.

Given a sequence $\left\{i_{n}\right\} \in\{1,2, \ldots, a\}^{\mathbb{Z}}$ and integers $l, m$ we define the cylinder

$$
\hat{C}_{l}^{m}\left(\left\{i_{n}\right\}, t\right)=\bigcap_{k=l}^{m} \hat{f}^{-k}\left(\hat{K}_{i_{k}}\right)
$$

or

$$
\hat{C}_{l}^{m}\left(i_{0}, i_{1}, \ldots, i_{m-l}, t\right)=\bigcap_{k=l}^{m} \hat{f}^{-k}\left(\hat{K}_{i_{k-l}}\right) .
$$

Let $\Sigma_{t}=\left\{\left\{i_{k}\right\} \mid \hat{C}_{l}^{m}\left(\left\{i_{k}\right\}, t\right) \neq \emptyset \forall l, m \in \mathbb{Z}\right\}$ and let $\rho_{t}: \Sigma_{t} \rightarrow \hat{\Lambda}_{t}$ be the natural identification of sequences in $\Sigma_{t}$ and points in $\hat{\Lambda}_{t}$. 


\subsection{The measure's dependence on the parameters}

The first step is to estimate how the measure $\hat{\mu}_{\mathrm{SBR}}^{t}$ changes with the parameter $t$. This is done by using that $\hat{\mu}_{n}^{t}$ converges weakly to $\hat{\mu}_{\mathrm{SBR}}^{t}$.

Let $L_{1}>0$. The measure $\hat{\mu}_{n}^{t}=\frac{1}{n} \sum_{k=0}^{n-1} \hat{\nu}_{\hat{V}^{\mathrm{u}}} \circ \hat{f}_{t}^{-k}$ converges weakly to $\hat{\mu}_{\mathrm{SBR}}^{t}$. Hence there is a number $n_{0}\left(t, L_{1}\right)$ such that for any cylinder of length $L_{1}$

$$
\frac{1}{2} \leq \frac{\hat{\mu}_{\mathrm{SBR}}^{t}\left(\hat{C}_{-L_{1}}^{0}\right)}{\hat{\mu}_{n}^{t}\left(\hat{C}_{-L_{1}}^{0}\right)} \leq 2,
$$

for all $n \geq n_{0}$. Since $n_{0}\left(t, L_{1}\right)$ is measurable with respect to $t$, Lusin's theorem implies that for any $\varepsilon_{1}>0$ there is a set $J_{1} \subset J_{0}$ with $\nu\left(J_{0} \backslash J_{1}\right)<\varepsilon_{1}$ and a number $n_{1}$ such that $n_{1}>n_{0}\left(t, L_{1}\right)$ for all $t \in J_{1}$.

For a fixed cylinder $\hat{C}_{-L_{1}}^{0}$, the measure $\hat{\mu}_{n_{0}}^{t}\left(\hat{C}_{-L_{1}}^{0}\right)$ depends continuously on $t$, because the measure $\hat{\mu}_{n_{0}}^{t}$ involves taking finitely many preimages with respect to $\hat{f}_{t}$ and these preimages depends continuously on $t$. It is therefore possible to partition $I$ into finitely many subintervals $I=\bigcup_{k=1}^{m} I_{k}$ such that when $t_{1}, t_{2} \in I_{k}$ then

$$
\frac{1}{2} \leq \frac{\hat{\mu}_{n_{0}}^{t_{1}}\left(\hat{C}_{-L_{1}}^{0}\right)}{\hat{\mu}_{n_{0}}^{t_{2}}\left(\hat{C}_{-L_{1}}^{0}\right)} \leq 2,
$$

for any cylinder of length $L_{1}$. This implies that

$$
\frac{1}{4} \leq \frac{\hat{\mu}_{\mathrm{SBR}}^{t_{1}}\left(\hat{C}_{-L_{1}}^{0}\right)}{\hat{\mu}_{\mathrm{SBR}}^{t_{2}}\left(\hat{C}_{-L_{1}}^{0}\right)} \leq 4,
$$

for any cylinder of length $L_{1}$, provided $t_{1}, t_{2} \in I_{k} \cap J_{1}$ for some $k$.

For each $I_{k}$, choose a $t_{k} \in I_{k} \cap J_{1}$ and a cylinder $\hat{C}_{-L_{1}}^{0}\left(\left\{x_{n}\left(t_{k}\right)\right\}, t_{k}\right)$ with $m_{k}=\hat{\mu}_{\mathrm{SBR}}^{t_{k}}\left(\hat{C}_{-L_{1}}^{0}\left(\left\{x_{n}\left(t_{k}\right)\right\}, t_{k}\right)>0\right.$. For every $t \in I_{k} \cap J_{1}$, define

$$
\hat{\Omega}_{0, t}=\hat{C}_{-L_{1}}^{0}\left(\left\{x_{n}\left(t_{k}\right)\right\}, t\right) .
$$

Then

$$
\hat{\mu}_{\mathrm{SBR}}^{t}\left(\hat{\Omega}_{0, t}\right) \geq \frac{1}{4} m_{k}
$$

for all $t \in I_{k} \cap J_{1}$.

\subsection{Entropy}

It will be necessary to control the number of cylinders and the measure of the cylinders. As already noted, the general theory gives that the entropy of the measure $\hat{\mu}_{\mathrm{SBR}}^{t}$, satisfy $\log \left(\gamma_{\min }\right) \leq h_{\hat{\mu}_{\mathrm{SBR}}^{t}} \leq \log \left(\gamma_{\max }\right)$

The Shannon-McMillan-Breiman Theorem implies that for $t \in J_{1} \cap I_{k}$ and $\varepsilon_{2}>0$ there is a constant $A(t)$ such that

$$
\begin{aligned}
& \hat{\mu}_{\mathrm{SBR}}^{t}\left(\bigcap _ { L > 0 } \left\{\hat{x} \mid \exists \hat{C}_{-L}^{0}\left(\left\{x_{k}\right\}, t\right) \ni \hat{x}\right.\right. \text { with } \\
& \left.\left.\quad A(t)\left(\gamma_{\max }+\varepsilon_{2}\right)^{-L}<\hat{\mu}_{\mathrm{SBR}}^{t}\left(\hat{C}_{-L}^{0}\right)<A(t)\left(\gamma_{\min }-\varepsilon_{2}\right)^{-L}\right\}\right)>1-\frac{1}{8} m_{k} .
\end{aligned}
$$


The constant $A(t)$ can be chosen so that almost surely this estimate is also valid for the conditional measures on the stable manifolds,

$$
\begin{aligned}
& \hat{\mu}_{\mathrm{SBR}}^{t}\left(\bigcap _ { L > 0 } \left\{\hat{x} \mid \exists \hat{C}_{-L}^{0}\left(\left\{x_{k}\right\}, t\right) \ni \hat{x}\right.\right. \text { with } \\
& \left.\left.\quad A(t)\left(\gamma_{\max }+\varepsilon_{2}\right)^{-L}<\hat{\mu}_{\mathrm{SBR}}^{t, \mathrm{~s}, x}\left(\hat{C}_{-L}^{0}\right)<A(t)\left(\gamma_{\min }-\varepsilon_{2}\right)^{-L}\right\}\right)>1-\frac{1}{8} m_{k} .
\end{aligned}
$$

Let $\hat{\Omega}_{\mathrm{SMB}, t}$ be the set whose measure is estimated above.

An application of Lusin's theorem shows that that given $\varepsilon_{3}>0$ there exists a set $J_{2} \subset J_{1}$ and numbers $A_{k}$ such that

$$
\begin{aligned}
& \nu\left(J_{2} \backslash J_{1}\right)<\varepsilon_{3}, \\
& A(t) \leq A_{k}, \text { whenever } t \in I_{k} \cap J_{2} .
\end{aligned}
$$

For all $t \in I_{k} \cap J_{2}$, define $\hat{\Omega}_{t}=\hat{\Omega}_{0, t} \cap \hat{\Omega}_{\mathrm{SMB}, t}$. The estimates (21) and (3) shows that $\hat{\mu}_{\mathrm{SBR}}^{t}\left(\hat{\Omega}_{t}\right) \geq \frac{1}{8} m_{k}$.

It follows by (3) that the number of cylinders of length $L$ in $\hat{\Omega}_{t}$ satisfy

$$
N_{t}(n) \leq A_{k}\left(\gamma_{\max }+\varepsilon_{2}\right)^{n} .
$$

Let $L_{2}>0$ and consider for each $t \in I_{k}$ the set of words of length $L_{2}+1$

$$
\mathcal{C}_{t}=\left\{x_{0}, x_{1}, \ldots, x_{L_{2}} \mid x_{i} \in\{1,2, \ldots, a\}, \hat{C}_{0}^{L_{2}}\left(x_{0}, x_{1}, \ldots, x_{L_{2}}, t\right) \neq \emptyset\right\} .
$$

The cylinders $\hat{C}_{0}^{L_{2}}\left(\left\{x_{k}\right\}, t\right)$ change continuously with $t$. The fact that the sets $\hat{K}_{i}$ have piecewise $C^{2}$ boundaries allow us to draw the following conclusion. There is a partition of $I_{k}$ into finitely many intervals $I_{k, l}\left(L_{2}\right)$ such that $\mathcal{C}_{t}=\mathcal{C}_{t^{\prime}}$ if $t^{\prime}, t \in I_{k, l}\left(L_{2}\right)$ for some $l$. Indeed, when $t$ runs over $I_{k}$, each cylinder appears and disappears only finitely many times. Let the finite set of $t$, for which some cylinder $\hat{C}_{0}^{L_{2}}\left(\left\{x_{k}\right\}, t\right)$ appears and disappears, define the endpoints of the intervals $I_{k, l}\left(L_{2}\right)$.

Any sequence in $\Sigma_{t} \cap \rho_{t}^{-1}\left(\hat{\Omega}_{t}\right)$ can be written as a concatenation of words from $\mathcal{C}_{t}$. Together with (44) this implies that for each $I_{k, l}$ the number of words of length $n$ in $\bigcup_{t \in I_{k, l} \cap J_{2}} \Sigma_{t} \cap \rho_{t}^{-1}\left(\hat{\Omega}_{t}\right)$ does not exceed $\left(A_{k}^{\frac{1}{L_{2}}}\left(\gamma_{\max }+\varepsilon_{2}\right)\right)^{n+L_{2}}$ for any $n$. Hence, we have the following lemma.

Lemma 2. For any $\varepsilon_{4}>0$ there is a number $L_{3}=L_{3}\left(\varepsilon_{4}\right)$ such that if $L_{2}>L_{3}$ then for each $I_{k, l}\left(L_{2}\right)$ the symbolic space $\Sigma_{I_{k, l}\left(L_{2}\right)}=\bigcup_{t \in I_{k, l}\left(L_{2}\right) \cap J_{2}} \Sigma_{t} \cap \rho_{t}^{-1}\left(\hat{\Omega}_{t}\right)$ satisfy

$$
N_{I_{k, l}\left(L_{2}\right)}(n) \leq B_{k, l}\left(\gamma_{\max }+\varepsilon_{2}+\varepsilon_{4}\right)^{n},
$$

for some $B_{k, l}$, where $N_{I_{k, l}\left(L_{2}\right)}(n)$ denotes the number of words of length $n$ in $\Sigma_{I_{k, l}\left(L_{2}\right)}$.

In order to make use of Lemma 2 choose $L_{2}>L_{3}$.

\subsection{Integrability of the densities}

The conditional measures of $\mu_{\mathrm{SBR}}^{t}$ on unstable manifolds are absolutely continuous with respect to Lebesgue measure. We will prove that the conditional 
measures on the stable manifolds are almost surely absolutely continuous with respect to Lebesgue measure. The local product structure of $\mu_{\mathrm{SBR}}^{t}$ then implies that $\mu_{\mathrm{SBR}}^{t}$ is absolutely continuous with respect to Lebesgue measure.

Take $x \in \Lambda=\pi(\hat{\Lambda})$ and let $W_{r}^{t, \mathrm{~s}}(y, x)=\left\{z \in W^{t, \mathrm{~s}}(x) \mid d(y, z) \leq r\right\}$. The derivative of $\mu_{\mathrm{SBR}}^{t, \mathrm{~s}, x}$ at $y$ is the limit

$$
D\left(\mu_{\mathrm{SBR}}^{t, \mathrm{~s}, x}, y\right)=\liminf _{r \rightarrow 0} \frac{\mu_{\mathrm{SBR}}^{t, \mathrm{~s}, x}\left(W_{r}^{t, \mathrm{~s}}(y, x)\right)}{2 r} .
$$

If the function $D\left(\mu_{\mathrm{SBR}}^{t, \mathrm{~s}, x}, y\right)$ is integrable on $W^{t, \mathrm{~s}}(x)$ then the measure $\mu_{\mathrm{SBR}}^{t, \mathrm{~s}, x}$ is absolutely continuous with respect to Lebesgue measure.

Let $k$ be fixed. We want to prove that for a.e. $t \in I_{k} \cap J_{2}$

$$
\int_{\Omega_{t}} \int_{\Omega_{t}} D\left(\left.\mu_{\mathrm{SBR}}^{t, \mathrm{~s}, x}\right|_{\Omega_{t}}, y\right) \mathrm{d} \mu_{\mathrm{SBR}}^{t, \mathrm{~s}, x}(y) \mathrm{d} \mu_{\mathrm{SBR}}^{t}(x)<\infty .
$$

This implies that the measure $\mu_{\mathrm{SBR}}^{t, \mathrm{~s}, x}$ restricted to the set $\Omega_{t}$ is absolutely continuous for a.e. $x \in \Omega_{t}$. Since the conditional measure on the unstable manifolds are absolutely continuous with respect to Lebesgue measure, this implies that $\left.\mu_{\mathrm{SBR}}^{t}\right|_{\Omega_{t}}$ is absolutely continuous with respect to Lebesgue measure. Since $\mu_{\mathrm{SBR}}^{t}\left(\Omega_{t}\right)>0$, ergodicity then implies that this also holds for the measure $\mu_{\mathrm{SBR}}^{t}$. Since $k$ is arbitrary this implies that $\mu_{\mathrm{SBR}}^{t}$ is absolutely continuous with respect to Lebesgue for a.e. $t \in I \cap J_{2}$.

Fatou's lemma implies that in order to prove (5) it suffices to prove that

$$
\liminf _{r \rightarrow 0} \frac{1}{r} \int_{\Omega_{t}} \int_{\Omega_{t}} \mu_{\mathrm{SBR}}^{t, \mathrm{~s}, x}\left(\Omega_{t} \cap W_{r}^{t, \mathrm{~s}}(y, x)\right) \mathrm{d} \mu_{\mathrm{SBR}}^{t, \mathrm{~s}, x}(y) \mathrm{d} \mu_{\mathrm{SBR}}^{t}(x)<\infty .
$$

We may rewrite this as

$$
\liminf _{r \rightarrow 0} \frac{1}{r} \int_{\Omega_{t}} \int_{\Omega_{t}} \int_{\Omega_{t}} \chi_{\left\{\left|y_{1}-z_{1}\right|<r\right\}} \mathrm{d} \mu_{\mathrm{SBR}}^{t, \mathrm{~s}, x}(z) \mathrm{d} \mu_{\mathrm{SBR}}^{t, \mathrm{~s}, x}(y) \mathrm{d} \mu_{\mathrm{SBR}}^{t}(x)<\infty .
$$

To prove that this holds for a.e. $t \in I_{k} \cap J_{2}$ we prove that for any $l$

$$
\begin{array}{r}
\liminf _{r \rightarrow 0} \frac{1}{r} \int_{I_{k, l} \cap J_{2}} \int_{\Omega_{t}} \int_{\Omega_{t}} \int_{\Omega_{t}} \chi_{\left\{\left|y_{1}-z_{1}\right|<r\right\}} \mathrm{d} \mu_{\mathrm{SBR}}^{t, \mathrm{~s}, x}(z) \mathrm{d} \mu_{\mathrm{SBR}}^{t, \mathrm{~s}, x}(y) \mathrm{d} \mu_{\mathrm{SBR}}^{t}(x) \mathrm{d} t \\
<\infty .
\end{array}
$$

This then implies that $\mu_{\mathrm{SBR}}^{t} \ll \nu$ for a.e. $t \in I_{k} \cap J_{2}$. Instead of proving (7) we use that $\mu_{\mathrm{SBR}}^{t}=\hat{\mu}_{\mathrm{SBR}}^{t} \circ \pi^{-1}$ and prove the equivalent condition

$$
\begin{array}{r}
\liminf _{r \rightarrow 0} \frac{1}{r} \int_{I_{k, l} \cap J_{2}} \int_{\hat{\Omega}_{t}} \int_{\hat{\Omega}_{t}} \int_{\hat{\Omega}_{t}} \chi_{\left\{\left|y_{1}-z_{1}\right|<r\right\}} \mathrm{d} \hat{\mu}_{\mathrm{SBR}}^{t, \mathrm{~s}, \hat{x}}(\hat{z}) \mathrm{d} \hat{\mu}_{\mathrm{SBR}}^{t, \mathrm{~s}, \hat{x}}(\hat{y}) \mathrm{d} \hat{\mu}_{\mathrm{SBR}}^{t}(\hat{x}) \mathrm{d} t \\
<\infty .
\end{array}
$$

Recall that $\rho_{t}: \Sigma_{t} \rightarrow \hat{\Lambda}_{t}$ maps sequences in $\Sigma_{t}$ to points in $\hat{\Lambda}_{t}$ in the natural way. Put $\mu_{\Sigma}^{t}=\hat{\mu}_{\mathrm{SBR}}^{t} \circ \rho_{t}$ and rewrite (8) as

$$
\begin{aligned}
\liminf _{r \rightarrow 0} \frac{1}{r} \int_{I_{k, l} \cap J_{2}} \int_{\hat{\Omega}_{t}} \int_{\rho_{t}^{-1}\left(\hat{\Omega}_{t}\right)} \int_{\rho_{t}^{-1}\left(\hat{\Omega}_{t}\right)} \chi_{\left\{\left|\rho\left(\left\{i_{n}\right\}\right)-\rho\left(\left\{j_{n}\right\}\right)\right|_{1}<r\right\}} & \mathrm{d} \mu_{\Sigma}^{t, \mathrm{~s}, \hat{x}}\left(\left\{i_{n}\right\}\right) \mathrm{d} \mu_{\Sigma}^{t, \mathrm{~s}, \hat{x}}\left(\left\{j_{n}\right\}\right) \mathrm{d} \hat{\mu}_{\mathrm{SBR}}^{t}(\hat{x}) \mathrm{d} t<\infty,
\end{aligned}
$$


where $|\cdots|_{1}$ denotes the difference in the first coordinate.

Embed all subshifts $\Sigma_{t}, t \in I_{k, l} \cap J_{1}$ into the larger subshift $\Sigma_{I_{k, l}}$ according to Lemma 2] The measures $\mu_{\Sigma}^{t}$ extend from $\Sigma_{t}$ to $\Sigma_{I_{k, l}}$ in a natural way since $\Sigma_{t}$ is a subset of $\Sigma_{I_{k, l}}$. A cylinder in $\Sigma_{I_{k, l}}$ will be denoted by

$$
{ }_{l}\left[\left\{i_{n}\right\}\right]_{m}={ }_{l}\left[i_{l} \cdots i_{m}\right]_{m}=\left\{\left\{j_{n}\right\} \in \Sigma_{I_{k, l}} \mid j_{n}=i_{n}, n=l, \ldots, m\right\} .
$$

To prove (9) we estimate the quantity

$$
\begin{aligned}
& T_{r}\left(\left\{\hat{\Omega}_{t} \mid t \in I_{k, l} \cap J_{2}\right\}\right)= \\
& \sum_{L>L_{2}} \sum_{\substack{-L\left[i_{-L}, \ldots, i_{0}\right]_{0} \\
\subset \Sigma_{I_{k, l}}}} \sum_{\substack{1 \leq l_{1}, l_{2} \leq a \\
l_{1} \neq l_{2}}} \int_{I_{k, l} \cap J_{2}} \int_{\hat{\Omega}_{t}} \int_{\rho_{t}^{-1}\left(\hat{\Omega}_{t}\right) \cap\left[l_{1}, i_{-L}, \ldots, i_{0}\right]} \int_{\rho_{t}^{-1}\left(\hat{\Omega}_{t}\right) \cap\left[l_{2}, i_{-L}, \ldots, i_{0}\right]} \\
& \quad \chi_{\left\{\left|\rho_{t}\left(\left\{i_{n}\right\}\right)-\rho_{t}\left(\left\{j_{n}\right\}\right)\right|_{1}<r\right\}} \mathrm{d} \mu_{\Sigma}^{t, \mathrm{~s}, \hat{x}}\left(\left\{j_{n}\right\}\right) \mathrm{d} \mu_{\Sigma}^{t, \mathrm{~s}, \hat{x}}\left(\left\{i_{n}\right\}\right) \mathrm{d} \hat{\mu}_{\mathrm{SBR}}^{t}(\hat{x}) \mathrm{d} t \quad(10)
\end{aligned}
$$

and show that $T_{r}<\eta r$ for all $r>0$ and some constant $\eta$. This implies (9) as follows. The product $\Sigma_{I_{k, l}} \times \Sigma_{I_{k, l}}$ can be written as

$$
\begin{aligned}
& \Sigma_{I_{k, l}} \times \Sigma_{I_{k, l}}=\bigcup_{L} \bigcup_{\substack{-L\left[i_{-L}, \ldots, i_{0}\right]_{0} \\
\subset \Sigma_{I_{k, l}}}} \bigcup_{\begin{array}{c}
1 \leq l_{1}, l_{2} \leq a \\
l_{1} \neq l_{2}
\end{array}} \\
& { }_{-L-1}\left[l_{1}, i_{-L}, \ldots, i_{0}\right]_{0} \times{ }_{-L-1}\left[l_{2}, i_{-L}, \ldots, i_{0}\right]_{0},
\end{aligned}
$$

i.e. $\Sigma_{I_{k, l}} \times \Sigma_{I_{k, l}}$ is the union over $L$ of the set of pair of sequences with the first $L$ letters equal. This implies that

$$
\begin{gathered}
\liminf _{r \rightarrow 0} \frac{1}{r} \int_{I_{k, l} \cap J_{2}} \int_{\hat{\Omega}_{t}} \int_{\rho_{t}^{-1}\left(\hat{\Omega}_{t}\right)} \int_{\rho_{t}^{-1}\left(\hat{\Omega}_{t}\right)} \chi_{\left\{\left|\rho\left(\left\{i_{n}\right\}\right)-\rho\left(\left\{j_{n}\right\}\right)\right|_{1}<r\right\}} \\
\mathrm{d} \mu_{\Sigma}^{t, \mathrm{~s}, \hat{x}}\left(\left\{i_{n}\right\}\right) \mathrm{d} \mu_{\Sigma}^{t, \mathrm{~s}, \hat{x}}\left(\left\{j_{n}\right\}\right) \mathrm{d} \hat{\mu}_{\mathrm{SBR}}^{t}(\hat{x}) \mathrm{d} t \\
=\liminf _{r \rightarrow 0} \frac{1}{r} T_{r}\left(\left\{\hat{\Omega}_{t} \mid t \in I_{k, l} \cap J_{2}\right\}\right),
\end{gathered}
$$

so (10) implies (9).

It remains to show (10). This will be done in 5.6. To do this, the estimate in 5.5 is needed.

\subsection{An estimate on power series}

The expression $\left|\rho_{t}\left(\left\{i_{n}\right\}\right)-\rho_{t}\left(\left\{j_{n}\right\}\right)\right|_{1}$ appearing in (10) can be expressed as a power series. The following estimate on this power series is an important part in proving (10).

If $\left(x_{1}, x_{2}, x_{3}\right) \in \hat{\Lambda}_{t}$ and $\left\{i_{n}\right\} \in \Sigma_{I_{k, l}}$ is the sequence such that $\rho_{t}\left(\left\{i_{n}\right\}\right)=$ $\left(x_{1}, x_{2}, x_{3}\right)$, then it is easy to see that

$$
x_{1}=\sum_{n=1}^{\infty} \prod_{l=1}^{n-1}\left(t \lambda_{i_{l-n}}\right) u_{i_{-n}}=\sum_{n=1}^{\infty} \prod_{l=1}^{n-1} \frac{\lambda_{i_{l-n}}}{\lambda_{\max }} u_{i_{-n}}\left(\lambda_{\max } t\right)^{n-1} .
$$

So the expression in the brackets of integrand in (10) can be rewritten in the form

$$
\left|\sum_{n=L+1}^{\infty}\left(\prod_{l=1}^{n-1} \lambda_{i_{l-n}} u_{i_{-n}}-\prod_{l=1}^{n-1} \lambda_{j_{l-n}} u_{j_{-n}}\right) t^{n-1}\right|<r .
$$


This is equivalent to

$$
\begin{aligned}
\mid 1+\sum_{n=L+2}^{\infty}\left(\prod_{l=1}^{n-1} \frac{\lambda_{i_{l-n}}}{\lambda_{\max }} u_{i_{-n}}-\prod_{l=1}^{n-1} \frac{\lambda_{j_{l-n}}}{\lambda_{\max }} u_{j_{-n}}\right) & \frac{\left(t \lambda_{\max }\right)^{n-1-L}}{u_{l_{1}}-u_{l_{2}}} \mid \\
& <\frac{r}{\left|u_{l_{1}}-u_{l_{2}}\right|} \frac{1}{\prod_{l=1}^{L}\left(t \lambda_{i_{l-n}}\right)},
\end{aligned}
$$

The coefficients of $\left(t \lambda_{\max }\right)^{n-1-L}$ in the sum in (12) are bounded by

$$
\begin{array}{r}
\max _{\left\{i_{n}\right\},\left\{j_{n}\right\}, n}\left(\prod_{l=1}^{n-1} \frac{\lambda_{i_{l-n}}}{\lambda_{\max }} u_{i_{-n}}-\prod_{l=1}^{n-1} \frac{\lambda_{j_{l-n}}}{\lambda_{\max }} u_{j_{-n}}\right) \\
\leq \frac{1}{u_{l_{1}}-u_{l_{2}}} \\
\leq \frac{\max \left\{\left|u_{i}-u_{j}\right|,\left|u_{i}\right|\right\}}{\min \left\{\left|u_{i}-u_{j}\right| \mid u_{i} \neq u_{j}\right\}}=: C,
\end{array}
$$

so an application of Corollary 2 with $C$ as above shows that

$$
\begin{aligned}
\mid\left\{t \lambda_{\max }|| \sum_{n=L}^{\infty}(\right. & \left.\left.\prod_{l=1}^{n-1} \lambda_{i_{l-n}} u_{i_{-n}}-\prod_{l=1}^{n-1} \lambda_{j_{l-n}} u_{j_{-n}}\right) t^{n-1} \mid<r\right\} \mid \\
& <\delta^{-1} \frac{\left(t_{0} \lambda_{\min }\right)^{-L}}{\min \left\{\left|u_{i}-u_{j}\right| \mid u_{i} \neq u_{j}\right\}} r=E \lambda_{\max }\left(t_{0} \lambda_{\min }\right)^{-L} r
\end{aligned}
$$

if $t_{1} \lambda_{\max }<\min \left\{\frac{1}{2 C}, 0.68\right\}$.

\subsection{The final step}

Put $F\left(t,\left\{i_{n}\right\},\left\{j_{n}\right\}\right)=\chi_{\left\{\left|\rho_{t}\left(\left\{i_{n}\right\}\right)-\rho_{t}\left(\left\{j_{n}\right\}\right)\right|_{1}<r\right\}}$ and rewrite (10) as

$$
\begin{array}{r}
T_{r}\left(\left\{\hat{\Omega}_{t} \mid t \in I_{k, l} \cap J_{2}\right\}\right)= \\
\sum_{L>L_{2}} \sum_{\substack{-L\left[i_{-L}, \ldots, i_{0}\right]_{0} \\
\subset \Sigma_{I_{k}, l}}} \sum_{\substack{1 \leq l_{1}, l_{2} \leq a \\
l_{1} \neq l_{2} \\
\left(I_{k, l} \cap J_{2}\right.}} \int_{\hat{\Omega}_{t}} \int_{\rho_{t}\left(\hat{\Omega}_{t}\right) \cap\left[l_{1}, i_{-L}, \ldots, i_{0}\right]} \int_{\rho_{t}\left(\hat{\Omega}_{t}\right) \cap\left[l_{2}, i_{-L}, \ldots, i_{0}\right]} \\
F\left(t,\left\{i_{n}\right\},\left\{j_{n}\right\}\right) \mathrm{d} \mu_{\Sigma}^{t, \mathrm{~s}, \hat{x}}\left(\left\{j_{n}\right\}\right) \mathrm{d} \mu_{\Sigma}^{t, \mathrm{~s}, \hat{x}}\left(\left\{i_{n}\right\}\right) \mathrm{d} \hat{\mu}_{\mathrm{SBR}}^{t}(\hat{x}) \mathrm{d} t . \quad(14)
\end{array}
$$

To estimate the quantity in (14) we want to change the order of integration to integrate with respect to $t$ first and then use the estimate (13). This can not be done immediately because the other integrals depends on $t$. To get around this problem the function $F\left(t,\left\{i_{n}\right\},\left\{j_{n}\right\}\right)$ will be bounded by a function $G\left(t,\left\{i_{n}\right\},\left\{j_{n}\right\}\right)$ which is constant on cylinders. More precisely

Lemma 3. For each pair of cylinders $\left[l_{1}, i_{-L}, \ldots, i_{0}\right]$ and $\left[l_{2}, i_{-L}, \ldots, i_{0}\right]$ appearing in (14) there is a partition into finitely many cylinders

$$
\left[l_{1}, i_{-L}, \ldots, i_{0}\right]=\bigcup_{p=1}^{n_{\alpha}} S_{\alpha}^{p},\left[l_{2}, i_{-L}, \ldots, i_{0}\right]=\bigcup_{q=1}^{n_{\beta}} S_{\beta}^{q}
$$

and functions $G_{S_{\alpha}^{p}, S_{\beta}^{q}}(t)$ with

$$
G_{S_{\alpha}^{p}, S_{\beta}^{q}}(t) \geq F\left(t,\left\{i_{n}\right\},\left\{j_{n}\right\}\right), \quad \forall t \in I_{k, l} \cap J_{1}, \forall\left\{i_{n}\right\} \in S_{\alpha}^{p}, \forall\left\{j_{n}\right\} \in S_{\beta}^{q}
$$


and

$$
\int_{I_{k, l}} G_{S_{\alpha}^{p}, S_{\beta}^{q}}(t) \mathrm{d} t<2 E \lambda_{\max }\left(t_{0} \lambda_{\min }\right)^{-L} r
$$

Proof. For fixed $\hat{y}$ and $\hat{z}$ the estimate (13) implies that

$$
\left|\left\{t \mid F\left(t,\left\{i_{n}\right\},\left\{j_{n}\right\}\right)>0\right\}\right|<E \lambda_{\max }\left(t_{0} \lambda_{\min }\right)^{-L} r
$$

and

$$
\left|\left\{t|| \rho_{t}\left(\left\{i_{n}\right\}\right)-\left.\rho_{t}\left(\left\{j_{n}\right\}\right)\right|_{1}<2 r\right\}\right|<2 E \lambda_{\max }\left(t_{0} \lambda_{\min }\right)^{-L} r .
$$

The function $\left(t,\left\{i_{n}\right\},\left\{j_{n}\right\}\right) \mapsto\left|\rho_{t}\left(\left\{i_{n}\right\}\right)-\rho_{t}\left(\left\{j_{n}\right\}\right)\right|_{1}$ depends continuously on $t$, $\left\{i_{n}\right\}$ and $\left\{j_{n}\right\}$. Choose $L^{\prime}$ so large that

$$
\frac{2 \max \left\{u_{i}-u_{j}\right\}\left(t_{1} \lambda_{\max }\right)^{L^{\prime}+3}}{1-t_{1} \lambda_{\max }}<r .
$$

The sets $\left[l_{1}, i_{-L}, \ldots, i_{0}\right]$ and $\left[l_{1}, i_{-L}, \ldots, i_{0}\right]$ can be partitioned into cylinders of length $L^{\prime}+L+3$

$$
\begin{aligned}
& {\left[l_{1}, i_{-L}, \ldots, i_{0}\right]=\bigcup_{p=1}^{n_{\alpha}} S_{\alpha}^{p}=\bigcup_{p=1}^{n_{\alpha}}\left[\alpha_{-L^{\prime}}(p), \ldots, \alpha_{0}(p), l_{1}, i_{-L}, \ldots, i_{0}\right],} \\
& {\left[l_{2}, i_{-L}, \ldots, i_{0}\right]=\bigcup_{q=1}^{n_{\beta}} S_{\beta}^{q}=\bigcup_{q=1}^{n_{\beta}}\left[\beta_{-L^{\prime}}(q), \ldots, \beta_{0}(q), l_{2}, i_{-L}, \ldots, i_{0}\right] .}
\end{aligned}
$$

Note that $n_{\alpha}$ and $n_{\beta}$ can be bounded uniformly by $n_{\alpha}, n_{\beta} \leq a^{L^{\prime}+1}$.

As in (11) the expression ||$\rho_{t}\left(\left\{i_{n}^{(1)}\right\}\right)-\left.\rho_{t}\left(\left\{j_{n}^{(1)}\right\}\right)\right|_{1}-\left|\rho_{t}\left(\left\{i_{n}^{(2)}\right\}\right)-\rho_{t}\left(\left\{j_{n}^{(2)}\right\}\right)\right|_{1} \mid$ can be written as

$$
\begin{aligned}
& \| \rho_{t}\left(\left\{i_{n}^{(1)}\right\}\right)-\left.\rho_{t}\left(\left\{j_{n}^{(1)}\right\}\right)\right|_{1}-\left|\rho_{t}\left(\left\{i_{n}^{(2)}\right\}\right)-\rho_{t}\left(\left\{j_{n}^{(2)}\right\}\right)\right|_{1} \mid||\left|\sum_{n=1}^{\infty}\left(\prod_{l=1}^{n-1} \lambda_{i_{l-n}^{(1)}} u_{i_{-n}^{(1)}}-\prod_{l=1}^{n-1} \lambda_{j_{l-n}^{(1)}} u_{j_{-n}^{(1)}}\right) t^{n-1}\right| \\
&-\mid \sum_{n=1}^{\infty}\left(\prod_{l=1}^{n-1} \lambda_{i_{l-n}^{(2)}} u_{i_{-n}^{(2)}}-\prod_{l=1}^{n-1} \lambda_{j_{l-n}^{(2)}} u_{j_{-n}^{(2)}}\right) t^{n-1} \|
\end{aligned}
$$

If $\left\{i_{n}^{(1)}\right\},\left\{i_{n}^{(2)}\right\} \in S_{\alpha}^{p},\left\{j_{n}^{(1)}\right\},\left\{j_{n}^{(2)}\right\} \in S_{\beta}^{q}$ for some $p$ and $q$, then since $\left\{i_{n}^{(1)}\right\}$ and $\left\{i_{n}^{(2)}\right\}$ respectively $\left\{j_{n}^{(1)}\right\}$ and $\left\{j_{n}^{(2)}\right\}$ are equal on the first $L^{\prime}+L+3$ letters, the first $L^{\prime}+L+3$ terms in this powerseries are zero. Hence

$$
\begin{aligned}
\| \rho_{t}\left(\left\{i_{n}^{(1)}\right\}\right)- & \left.\rho_{t}\left(\left\{j_{n}^{(1)}\right\}\right)\right|_{1}-\left|\rho_{t}\left(\left\{i_{n}^{(2)}\right\}\right)-\rho_{t}\left(\left\{j_{n}^{(2)}\right\}\right)\right|_{1} \mid= \\
= & \| \sum_{n=L+L^{\prime}+4}^{\infty}\left(\prod_{l=1}^{n-1} \lambda_{i_{l-n}^{(1)}} u_{i_{-n}^{(1)}}-\prod_{l=1}^{n-1} \lambda_{j_{l-n}^{(1)}} u_{j_{-n}^{(1)}}\right) t^{n-1} \mid \\
& -\mid \sum_{n=L+L^{\prime}+4}^{\infty}\left(\prod_{l=1}^{n-1} \lambda_{i_{l-n}^{(2)}} u_{i_{-n}^{(2)}}-\prod_{l=1}^{n-1} \lambda_{j_{l-n}^{(2)}} u_{j_{-n}^{(2)}}\right) t^{n-1} \| \\
\leq & \sum_{n=L+L^{\prime}+4}^{\infty} 2 \max \left\{u_{i}-u_{j}\right\}\left(\lambda_{\max } t\right)^{n-1} \\
\leq & \frac{2 \max \left\{u_{i}-u_{j}\right\}\left(\lambda_{\max } t_{1}\right)^{L+L^{\prime}+3}}{1-\lambda_{\max } t_{1}}<r .
\end{aligned}
$$


The last inequality follows from the choice of $L^{\prime}$ that $\frac{2 \max \left\{u_{i}-u_{j}\right\}\left(t_{1} \lambda_{\max }\right)^{L^{\prime}+3}}{1-t_{1} \lambda_{\max }}<$ $r$.

For each pair $S_{\alpha}^{p}$ and $S_{\beta}^{q}$, take $\left\{i_{n}^{(p)}\right\} \in S_{\alpha}^{p}$ and $\left\{j_{n}^{(q)}\right\} \in S_{\beta}^{q}$. Put

$$
G_{S_{\alpha}^{p}, S_{\beta}^{q}}(t)=\chi_{\left\{\left|\rho_{t}\left(\left\{i_{n}^{(p)}\right\}\right)-\rho_{t}\left(\left\{j_{n}^{(q)}\right\}\right)\right|_{1}<2 r\right\}} .
$$

Then the estimates (16) and (17) imply that

$$
G_{S_{\alpha}^{p}, S_{\beta}^{q}}(t) \geq F\left(t,\left\{i_{n}\right\},\left\{j_{n}\right\}\right), \forall t \in I_{k, l} \cap J_{1}, \forall\left\{i_{n}\right\} \in S_{\alpha}^{p}, \forall\left\{j_{n}\right\} \in S_{\beta}^{q}
$$

and

$$
\int_{I_{k, l}} G_{S_{\alpha}^{p}, S_{\beta}^{q}}(t) \mathrm{d} t<2 E \lambda_{\max }\left(t_{0} \lambda_{\min }\right)^{-L} r
$$

Lemma 3 is used to estimate the integrals in (14) in the following way.

$$
\begin{aligned}
& \mathcal{I}:= \int_{I_{k, l} \cap J_{2}} \int_{\hat{\Omega}_{t}} \int_{\rho_{t}^{-1}\left(\hat{\Omega}_{t}\right) \cap\left[l_{1}, i_{-L}, \ldots, i_{0}\right]} \int_{\rho_{t}^{-1}\left(\hat{\Omega}_{t}\right) \cap\left[l_{2}, i_{-L}, \ldots, i_{0}\right]} \\
&= \sum_{p, q} \int_{I_{k, l} \cap J_{2}} \int_{\hat{\Omega}_{t}} \int_{\rho_{t}^{-1}\left(\hat{\Omega}_{t}\right) \cap S_{\alpha}^{p}} \int_{\rho_{t}^{-1}\left(\hat{\Omega}_{t}\right) \cap S_{\beta}^{q}} \\
& F_{L, i_{-L}, \cdots, i_{0}}\left(t,\left\{i_{n}\right\},\left\{j_{n}\right\}\right) \mathrm{d} \mu_{\Sigma}^{t, \mathrm{~s}, \hat{x}}\left(\left\{j_{n}\right\}\right) \mathrm{d} \mu_{\Sigma}^{t, \mathrm{~s}, \hat{x}}\left(\left\{i_{n}\right\}\right) \mathrm{d} \hat{\mu}_{\mathrm{SBR}}^{t}(\hat{x}) \mathrm{d} t \\
& \leq \sum_{p, q} \int_{I_{k, l} \cap J_{2}} \int_{\hat{\Omega}_{t}} \int_{\rho_{t}^{-1}\left(\hat{\Omega}_{t}\right) \cap S_{\alpha}^{p}} \int_{\rho_{t}^{-1}\left(\hat{\Omega}_{t}\right) \cap S_{\beta}^{q}} G_{S_{\alpha}^{p}, S_{\beta}^{q}}(t) \mathrm{d} \mu_{\Sigma}^{t, \mathrm{~s}, \hat{x}}\left(\left\{j_{n}\right\}\right) \mathrm{d} \mu_{\Sigma}^{t, \mathrm{~s}, \hat{x}}\left(\left\{i_{n}\right\}\right) \mathrm{d} \hat{\mu}_{\mathrm{SBR}}^{t}(\hat{x}) \mathrm{d} t \\
&= \sum_{p, q} \int_{I_{k, l} \cap J_{2}} \int_{\hat{\Omega}_{t}} G_{S_{\alpha}^{p}, S_{\beta}^{q}}(t) \\
& \mu_{\Sigma}^{t, \mathrm{~s}, \hat{x}}\left(\rho_{t}^{-1}\left(\hat{\Omega}_{t}\right) \cap S_{\alpha}^{p}\right) \mu_{\Sigma}^{t, \mathrm{~s}, \hat{x}}\left(\rho_{t}^{-1}\left(\hat{\Omega}_{t}\right) \cap S_{\beta}^{q}\right) \mathrm{d} \hat{\mu}_{\mathrm{SBR}}^{t}(\hat{x}) \mathrm{d} t .
\end{aligned}
$$

This can be estimated by

$$
\begin{aligned}
\mathcal{I} \leq \max _{p, q}( & \left(\int_{I_{k, l} \cap J_{1}} G_{S_{\alpha}^{p}, S_{\beta}^{q}}(t) \mathrm{d} t\right) \\
& \sup _{t}\left(\sum_{p, q} \int_{\hat{\Omega}_{t}} \mu_{\Sigma}^{t, \mathrm{~s}, \hat{x}}\left(\rho_{t}^{-1}\left(\hat{\Omega}_{t}\right) \cap S_{\alpha}^{p}\right) \mu_{\Sigma}^{t, \mathrm{~s}, \hat{x}}\left(\rho_{t}^{-1}\left(\hat{\Omega}_{t}\right) \cap S_{\beta}^{q}\right) \mathrm{d} \hat{\mu}_{\mathrm{SBR}}^{t}(\hat{x})\right) .
\end{aligned}
$$

The maximum is estimated by (15) and the sum can be eliminated using that $\left\{S_{\alpha}^{p}\right\}$ and $\left\{S_{\beta}^{q}\right\}$ are partitions of the cylinders $\left[l_{1}, i_{-L}, \ldots, i_{0}\right]$ and $\left[l_{1}, i_{-L}, \ldots, i_{0}\right]$ respectively:

$$
\begin{aligned}
\mathcal{I} \leq 2 E \lambda_{\max }\left(t_{0} \lambda_{\min }\right)^{-L} r \sup _{t}( & \int_{\hat{\Omega}_{t}} \mu_{\Sigma}^{t, \mathrm{~s}, \hat{x}}\left(\rho_{t}^{-1}\left(\hat{\Omega}_{t}\right) \cap\left[l_{1}, i_{-L}, \ldots, i_{0}\right]\right) \\
& \left.\mu_{\Sigma}^{t, \mathrm{~s}, \hat{x}}\left(\rho_{t}^{-1}\left(\hat{\Omega}_{t}\right) \cap\left[l_{2}, i_{-L}, \ldots, i_{0}\right]\right) \mathrm{d} \hat{\mu}_{\mathrm{SBR}}^{t}(\hat{x})\right) .
\end{aligned}
$$


The measure of the cylinders is estimated with (3).

$$
\begin{aligned}
\mathcal{I} & \leq 2 E \lambda_{\max }\left(t_{0} \lambda_{\min }\right)^{-L} \sup _{t}\left(\int_{\hat{\Omega}_{t}} A_{k}^{2}\left(\gamma_{\min }-\varepsilon_{2}\right)^{-2(L+1)} \mathrm{d} \hat{\mu}_{\mathrm{SBR}}^{t}(\hat{x})\right) \\
& \leq 2 E \lambda_{\max }\left(t_{0} \lambda_{\min }\right)^{-L} r A_{k}^{2}\left(\gamma_{\min }-\varepsilon_{2}\right)^{-2(L+1)} .
\end{aligned}
$$

Thus

$$
\begin{aligned}
& T_{r}\left(\left\{\hat{\Omega}_{t} \mid t \in I_{k, l} \cap J_{2}\right\}\right) \\
& \leq \sum_{L=L_{2}}^{\infty} \sum_{\underline{i}} \sum_{\substack{l=1 \\
m}} \sum_{\substack{1 \leq l_{1}, l_{2} \leq a \\
l_{1} \neq l_{2}}} 2 \operatorname{Er}\left(t_{0} \lambda_{\min }\right)^{-L} A_{k}^{2}\left(\gamma_{\min }-\varepsilon_{2}\right)^{-2(L+1)} \\
& \leq \sum_{L=L_{2}}^{\infty} 2 E r B_{k, l}\left(\gamma_{\max }+\varepsilon_{2}+\varepsilon_{4}\right)^{L} m\left(a^{2}-a\right)\left(t_{0} \lambda_{\min }\right)^{-L} A_{k}^{2}\left(\gamma_{\min }-\varepsilon_{2}\right)^{-2 L} \\
& =\sum_{L=L_{2}}^{\infty} 2 E A_{k}^{2} B_{k, l} m\left(a^{2}-a\right) r\left(\frac{\gamma_{\max }+\varepsilon_{2}+\varepsilon_{4}}{t_{0} \lambda_{\min }\left(\gamma_{\min }-\varepsilon_{2}\right)^{2}}\right)^{L} .
\end{aligned}
$$

If $\frac{t_{0} \lambda_{\min } \gamma_{\min }^{2}}{\gamma_{\max }}>1$ then it is possible to choose $\varepsilon_{2}$ and $\varepsilon_{4}$ so small that

$$
\tau=\frac{\gamma_{\max }+\varepsilon_{2}+\varepsilon_{4}}{t_{0} \lambda_{\min }\left(\gamma_{\min }-\varepsilon_{2}\right)^{2}}<1 .
$$

Then $T_{r} \leq 2 E A_{k}^{2} B_{k, l} m\left(a^{2}-a\right) \tau^{L_{2}} \frac{1}{1-\tau} r$. This implies that $\mu_{\mathrm{SBR}}^{t}$ is absolutely continuous with respect to Lebesgue measure for a.e. $t \in I \cap J_{2}$. Let $\varepsilon_{0}, \varepsilon_{1}, \varepsilon_{3} \rightarrow$ 0 . Then $\nu\left(I \cap J_{2}\right)>\nu(I)-\varepsilon_{0}-\varepsilon_{1}-\varepsilon_{3} \rightarrow \nu(I)$ and this shows that $\hat{\mu}_{\mathrm{SBR}}^{t}$ is absolutely continuous with respect to Lebesgue measure for a.e. $t \in I_{k}$.

\section{Application to fat Belykh maps}

The Belykh maps are defined as follows. Let $-1<k<1$ and put $K=[-1,1]^{2}$, $K_{1}=\left\{\left(x_{1}, x_{2}\right) \in(-1,1)^{2} \mid x_{2}>k x_{1}\right\}$ and $K_{2}=\left\{\left(x_{1}, x_{2}\right) \in(-1,1)^{2} \mid x_{2}<\right.$ $\left.k x_{1}\right\}$. The discontinuity set is $N=\left\{\left(x_{1}, x_{2}\right) \mid x_{2}=k x_{1}\right\}$ and the border is $M=$ $\left\{\left(x_{1}, x_{2}\right) \in K \mid x_{1}= \pm 1\right.$ or $\left.x_{2}= \pm 1\right\}$. The Belykh maps $f_{\lambda, \gamma, k}: K_{1} \cup K_{2} \rightarrow K$ are defined by

$$
\begin{aligned}
& \left.f_{\lambda, \gamma, k}\right|_{K_{1}}\left(x_{1}, x_{2}\right)=\left(\lambda x_{1}+(1-\lambda), \gamma x_{2}-(\gamma-1)\right), \\
& \left.f_{\lambda, \gamma, k}\right|_{K_{2}}\left(x_{1}, x_{2}\right)=\left(\lambda x_{1}-(1-\lambda), \gamma x_{2}+(\gamma-1)\right),
\end{aligned}
$$

where $0<\lambda<1$ and $1<\gamma \leq \frac{2}{1+|k|}$.

These maps were first introduced for $\lambda<\frac{1}{2}$ by Belykh in [2] as a model of a Poincaré map from phase synchronisation. It was investigated in the case $\lambda<\frac{1}{2}$ in [14 and 15. Schmeling and Troubetzkoy studied in 17] the Belykh maps when $\lambda>\frac{1}{2}$ and called this case the fat Belykh map.

The Belykh maps satisfy the conditions (A1) and (A2). Here

$$
C=1 \text { and } C<f_{4}(x) \text { if } x<Q_{4}=0.61
$$

and we conclude:

Theorem 2. Let $P=\{(\lambda, \gamma, k) \mid \gamma \lambda>1, \lambda<0.61\}$. For Lebesgue almost all $(\gamma, \lambda, k) \in P$ the fat Belykh map $f_{\lambda, \gamma, k}$ has an absolutely continuous invariant measure. 


\section{Decay of correlations}

Applying Young's scheme from [23] Chernov proved in [7] the exponential decay of correlations for Hölder continuous functions for a class of piecewise hyperbolic systems with singularities in arbitrary dimensions. This result can be used in the following way.

Let $H_{\eta}=\left\{\phi: K \rightarrow \mathbb{R}|\exists C:| \phi(x)-\phi(y) \mid \leq C d(x, y)^{\eta}, \forall x, y \in K\right\}$ be the set of Hölder continuous functions on $K$.

Theorem 3. Assume that $f: K \rightarrow K$ satisfies the assumptions (A1) and (A2) and assume that $\left(f^{n}, \mu_{\mathrm{SBR}}\right)$ is ergodic for every $n \geq 1$. For every $\eta>0$ there exists a constant $\theta \in(0,1)$ such that for every $\phi, \psi \in H_{\eta}$ there is a constant $C(\phi, \psi)$ such that

$$
\left|\int_{K} \phi \circ f^{n} \cdot \psi \mathrm{d} \mu_{\mathrm{SBR}}-\int_{K} \phi \mathrm{d} \mu_{\mathrm{SBR}} \int_{K} \psi \mathrm{d} \mu_{\mathrm{SBR}}\right| \leq C \theta^{n}
$$

for all $n \in \mathbb{N}$.

Proof. Lift $f: K \rightarrow K$ to $\hat{f}: \hat{K} \rightarrow \hat{K}$ as in Section [5.1. The lift $\hat{f}$ is just a model for the natural extension of $f$. The natural extension is ergodic if and only if the original system is ergodic, see Theorem 1 in Chapter $10, \S 4$ of [8]. Hence $\left(\hat{f}^{n}, \hat{\mu}_{\mathrm{SBR}}\right)$ is ergodic for every $n \geq 1$.

The function $\phi: K \rightarrow \mathbb{R}$ is lifted to $\hat{\phi}: \hat{K} \rightarrow \mathbb{R}$ by $\hat{\phi}=\phi \circ \pi$ and $\hat{\psi}$ is lifted in the same way.

Note that $\hat{\phi}, \hat{\psi} \in \hat{H}_{\eta}=\left\{\hat{\phi}: \hat{K} \rightarrow \mathbb{R}|\exists C:| \hat{\phi}(x)-\hat{\phi}(y) \mid \leq C d(x, y)^{\eta}, \forall x, y \in\right.$ $\hat{K}\}$ and

$$
\begin{aligned}
& \int_{K} \phi \circ f^{n} \cdot \psi \mathrm{d} \mu_{\mathrm{SBR}}=\int_{\hat{K}} \hat{\phi} \circ \hat{f}^{n} \cdot \hat{\psi} \mathrm{d} \hat{\mu}_{\mathrm{SBR}}, \\
& \int_{K} \phi \mathrm{d} \mu_{\mathrm{SBR}}=\int_{\hat{K}} \hat{\phi} \mathrm{d} \hat{\mu}_{\mathrm{SBR}}, \int_{K} \psi \mathrm{d} \mu_{\mathrm{SBR}}=\int_{\hat{K}} \hat{\psi} \mathrm{d} \hat{\mu}_{\mathrm{SBR}} .
\end{aligned}
$$

Theorem 1.1 in [7] states that $\left(\hat{f}, \hat{\mu}_{\mathrm{SBR}}\right)$ has exponential decay of correlations so this implies that $\left(f, \mu_{\mathrm{SBR}}\right)$ has exponential decay of correlations. 


\section{References}

[1] J. C. Alexander, J. A. Yorke, Fat baker's transformations, Ergod. Th. \& Dynam. Sys. 4 (1984), 1-23

[2] V. P. Belykh, Models of discrete systems of phase synchronization, Systems of Phase Synchronization. Eds. V. V. Shakhildyan and L. N. Belynshina. Radio i Svyaz, Moscow, 1982, 61-176

[3] J. Buzzi, Intrinsic ergodicity of affine maps in $[0,1]^{d}$, Monatsh. Math., 124 (1997), 97-118

[4] J. Buzzi, Absolutely continuous invariant measures for generic multidimensional piecewise affine expanding maps, Internat. J. Bifur. Chaos Appl. Sci. Engrg. 9 (1999), 1743-1750

[5] J. Buzzi, Absolutely continuous invariant probability measures for arbitrary expanding piecewise $\mathbb{R}$-analytic mappings of the plane, Ergodic Theory Dynam. Systems 20 (2000), 697-708

[6] J. Buzzi, G Keller, Zeta functions and transfer operators for multidimensional piecewise affine and expanding maps, Ergodic Theory Dynam. Systems 21 (2001), 689-716

[7] N. Chernov, Statistical properties of piecewise smooth hyperbolic systems in high dimensions, Discrete Contin. Dynam. Systems 5 (1999), 425-448

[8] I. P. Cornfeld, S. V. Fomin, Ya. G. Sinaŭ, Ergodic Theory, Grundlehren der Mathematischen Wissenschaften 245, Springer-Verlag, New York, 1982, ISBN 0-387-90580-4

[9] A. Katok, J.-M. Strelcyn, Invariant manifolds, entropy and billiards; smooth maps with singularities, Lecture Notes in Mathematics 1222, Springer-Verlag, Berlin, 1986, ISBN 3-540-17190-8

[10] B. Kruglikov, M. Rypdal, Entropy via multiplicity, ArXiv:math.DS/0505019

[11] J. Neunhäuserer, Dimension theoretical properties of generalized Baker's transformations, Nonlinearity 15, 1299-1307, 2002

[12] Y. Peres, B. Solomyak, Absolute continuity of Bernoulli convolutions, a simple proof, Math. Research Letters 3 (1996), 231-236

[13] T. Persson, A piecewise hyperbolic map with absolutely continuous invariant measure, Preprints in Mathematical Sciences 2005:29, LUTFMA-50652005, ISSN 1403-9338

[14] Ya. B. Pesin, Dynamical systems with generalized hyperbolic attractors: hyperbolic, ergodic and topological properties, Ergod. Th. \& Dynam. Sys. 12 (1992), 123-151

[15] E. A. Sataev, Invariant measures for hyperbolic maps with singularities, Russian Math. Surveys 47 (1992), 191-251 
[16] J. Schmeling, A dimension formula for endomorphisms - the Belykh family, Ergod. Th. \& Dynam. Sys. 18 (1998), 1283-1309

[17] J. Schmeling, S. Troubetzkoy, Dimension and invertibility of hyperbolic endomorphisms with singularities, Ergod. Th. \& Dynam. Sys. 18 (1998), $1257-1282$

[18] B. Solomyak, On the random series $\sum \pm \lambda^{i}$ (an Erdös problem), Annals of Math. 142 (1995), 611-625

[19] M. Tsujii, Absolutely continuous invariant measures for piecewise realanalytic expanding maps on the plane, Comm. Math. Phys. 208 (2000), 605-622

[20] M. Tsujii, Absolutely continuous invariant measures for expanding piecewise linear maps, Invent. Math. 143 (2001), 349-373

[21] M. Tsujii, Fat solenoidal attractors, Nonlinearity 14 (2001), 1011-1027

[22] M. Tsujii, Physical measures for partially hyperbolic surface endomorphisms, to appear in Acta Mathematica

[23] L.-S. Young, Statistical properties of dynamical systems with some hyperbolicity, Annals of Math. 147 (1998), 585-650 